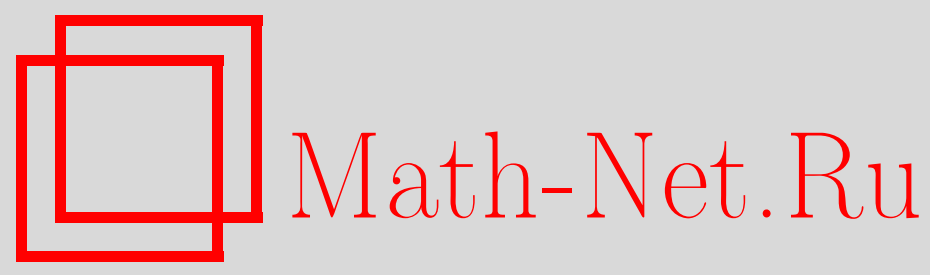

В. А. Антонов, И. А. Тюрина, А. П. Ческидов, Группы с малыми централизаторами, Матем. заметки, 2001, том 69, выпуск 5, 643-655

DOI: https://doi.org/10.4213/mzm528

Использование Общероссийского математического портала Math-Net.Ru подразумевает, что вы прочитали и согласны с пользовательским соглашением http://www . mathnet.ru/rus/agreement

Параметры загрузки:

IP : 34.229 .108 .108

26 апреля 2023 г., 13:14:57

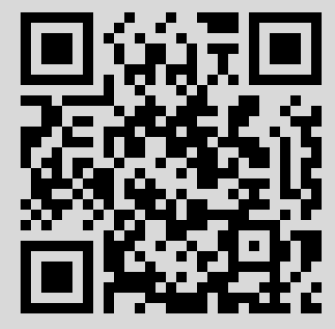


УДК 512.54

\title{
ГРУППЫ С МАЛЫМИ ЦЕНТРАЛИЗАТОРАМИ
}

\author{
В. А. Антонов, И. А. Тюрина, А. П. Ческидов
}

\begin{abstract}
Через $w(n)$ условимся обозначать количество множителей в представлении натурального числа $n$ в виде произведения простых чисел. Если $H$ - подгруппа конечной группы $G$, то $w(H)=w(|H|)$ и $v(G)=\max \{w(C(g)) \mid g \in G \backslash Z(G)\}$. В данной работе приводится полное описание групп без центра с условием $v(G)=4$.

Библиография: 12 названий.
\end{abstract}

Через $w(n)$ условимся обозначать количество множителей в представлении натурального числа $n$ в виде произведения простых чисел. Если $H$ - подгруппа конечной группы $G$, то $w(H)=w(|H|)$ и $v(G)=\max \{w(C(g)) \mid g \in G \backslash Z(G)\}$.

В работах [1] и [2] описаны конечные неабелевы групшы с условиями $v(G)=2$ и $v(G)=3$ соответственно. В предлагаемой работе исследуются группы без центра с условием $v(G)=4$.

Напомним [3], что М-группой называется неабелева групша, все собственные централизаторы в которой абелевы.

1. Неразрешимые группы. Обозначим через $l(p)$ число

$$
l(p)= \begin{cases}\max \left\{w(p-1), w\left(\frac{p+1}{2}\right)\right\}, & \text { если } p \equiv 1(4), \\ \max \left\{w(p+1), w\left(\frac{p-1}{2}\right)\right\}, & \text { если } p \equiv-1(4) .\end{cases}
$$

Tеорема 1.1. В конечной простой әруппе $G$ в том и только том случае выполняется условие $v(G)=4$, когда либо $G \cong A_{7}$, либо $G \cong P S L(2, q)$, где $q \in\left\{2^{4}, 7^{3}\right.$, $\left.5^{2}, p\right\}, p$ - простое число со свойством $l(p)=4$.

ДоКАЗАТЕЛЬСТво. Если $G$ является $\mathfrak{M - г р у п п о и ̆ , ~ т о ~ в ~ с и л у ~ с л е д с т в и я ~} 5.10$ из [3] $G \cong P S L(2,16)$. Пусть $G$ не $\mathfrak{M}$-группа. Так как $v(G)=4$, то любой собственньй централизатор в группе $G$ является либо абелевой группой, либо М-группой. Простые группы с таким свойством исследованы А. В. Васильевой [4]. Они исчерпьваются группами $P S L(2, q), q>3, S z\left(2^{2 n+1}\right), P S L(3, q), q>2, P S U\left(3, q^{2}\right), q>2, J_{1}, M_{11}$ и $A_{7}$. Группы $S z\left(2^{2 n+1}\right), P S L(3, q), q>2, P S U\left(3, q^{2}\right), q>2, J_{1}$ и $M_{11}$ не удовлетворяют условию $v(G)=4$.

Пусть $G \cong P S L\left(2, p^{n}\right)$. Так как $G$ не $\mathfrak{M - г р у п п а , ~ т о ~} p>2$ и $p^{n} \neq 5$. Максимальные централизаторы групш $P S L\left(2, p^{n}\right)$ исчерпываются силовскими $p$-подгрупшами, циклическими подгрупшами порядка $\left(p^{n}+1\right) / 2$, если $p^{n} \equiv 1(4)\left(\left(p^{n}-1\right) / 2\right.$, если $\left.p^{n} \equiv-1(4)\right)$, 
и группами диэдра порядка $\left(p^{n}-1\right)$ и $\left(p^{n}+1\right)$ соответственно. Поэтому $n<4$ и при $n=1$ должно вьполняться равенство $l(p)=4$.

Если $n=2$, то $p^{2}-1=8 \cdot\left(p^{2}-1\right) / 8$. Так как $v(P S L(2,9))=3$, то $p \neq 3$. А если $p>5$, то $w\left(p^{2}-1\right)>4$. Поэтому $p=5$.

Предположим теперь, что $n=3$. И пусть $p^{3} \equiv 1(4)$. Тогда

$$
p^{3}-1=4 \cdot \frac{p-1}{4}\left(p^{2}+p+1\right) .
$$

Если $p \equiv 1(3)$, то $w\left(p^{3}-1\right)>4$. Поэтому $p \equiv-1(3)$. Но тогда $p+1$ и $p^{2}-p+1$ делятся на 3 , т.е.

$$
\frac{p^{3}+1}{2}=9 \cdot \frac{p+1}{6} \cdot \frac{p^{2}-p+1}{3} .
$$

Если $p=5$, то $w\left(p^{3}-1\right)=w\left(\left(p^{3}+1\right) / 2\right)=3$, т.е. $p>5$. Так как $\left|P S L\left(2, p^{3}\right)\right|=p^{3}\left(p^{6}-1\right) / 2$, то $|G|$ делится на 7 . Если $(p-1) / 4=7$, то $p=29$. Но тогда $w\left(\left(p^{3}+1\right) / 2\right)>4$. Случай $(p+1) / 2=7$ невозможен, как невозможны и случаи $p^{2}+p+1=7$ или $\left(p^{2}-p+1\right) / 3=7$, т.е. случай $p^{3} \equiv 1(4)$ невозможен. Рассматривая аналогично случай $p^{3} \equiv-1(4)$, получим, что $p=7$.

Пользуясь случаем, отметим, что при доказательстве теоремы 4.1 из [2] были утеряны группы $P S L\left(2,3^{3}\right)$ и $P S L\left(2,5^{3}\right)$, удовлетворяющие условию $v(G)=3$.

ТЕОрема 1.2. В конечной непростой неразрешимой әруппе $G$ с тривиальным иентром в том и только том случае выполняется условие $v(G)=4$, когда $G$ одна из следующих әрупп:

1) $G \cong P G L(2, q), q \in\left\{5^{3}, 3^{3}, 5^{2}, 3^{2}, p\right\}$, где $p$ - простое число со свойством $l(p)=3$

2) $G \cong P S L(2, q) \lambda\langle\tau\rangle$, әде $q \in\left\{2^{3}, 3^{2}, 3^{3}\right\}, \tau$ - нетождественный автоморфизм поля $G F(q)$;

3) G обладает нормальной самоцентрализуемой әлементарной абелевой подәруппой $H$ порядка $5^{3}, G / H \cong P S L(2,5)$ и между множсествами неединичных ииклических подгрупп групп $H$ u $G / H$ существует такое взаимно однозначное соответствие $\varphi$, что $C(a) / H=\langle a\rangle^{\varphi}$ для всех $a \in H \backslash 1$;

4) $G=H \lambda P S L(2,5), H$ - әлементарная абелева группа порядка $p^{3}, p \equiv \pm 1(10)$, и $\operatorname{PSL}(2,5)$ действует на $H$ естественным образом (вложсение PSL $(2,5)$ в группу $G L(3, p)$ при $p \equiv \pm 1(10)$ в явном виде описано в работе [5]).

ДокАЗАТЕЛЬСТво. Пусть $G$ - группа из условия теоремы, $H$ - минимальный нормальный делитель группы $G$.

Предположим сначала, что $H$ неабелев. Тогда $H-$ простая группа и $v(H) \leqslant 4$. В силу [1], [2], теоремы 1.1 и замечания, сделанного после этой теоремы, $H$ изоморфна одной из групп $P S L(2, q), q \in\left\{2^{4}, 7^{3}, 5^{3}, 3^{3}, 2^{3}, 5^{2}, 3^{2}, p\right\}$, где $p$ - простое число со свойством $l(p) \leqslant 4$, или $A_{7}$.

Так как $v(G)=4$, то $H$ - единственньй минимальный нормальный делитель группы $G$ и $C(H)=1$. Но тогда $G \leqslant \operatorname{Aut}(H)$. Из $\operatorname{Aut}\left(A_{7}\right)=S_{7}$ и $v\left(A_{7}\right)=5$ следует, что $H ¥ A_{7}$. Как известно, $\operatorname{Aut}\left(P S L\left(2, p^{n}\right)\right)=P G L\left(2, p^{n}\right) \lambda\langle\tau\rangle$, где $\tau$ - автоморфизм поля, $|\tau|=n$. 
Если $H=P S L(2,16)$, то из $|\operatorname{Aut}(H)|=4|H|$ следует,что $\left|S_{2}(G)\right|>2^{4}$, что невозможно. Пусть $H=P S L(2,9)$. Так как $\left|S_{2}(\operatorname{Aut}(H))\right|=32$, то $G<\operatorname{Aut}(H)$. Поэтому либо $G=P G L(2,9)$, либо $G=P S L(2,9) \lambda\langle\tau\rangle$. Аналогично, если $H=P S L(2,25)$, то $G<\operatorname{Aut}(H)$. Если $\tau \in G$, то $C(\tau) \geqslant\langle\tau\rangle \times P S L(2,5)$, т.е. $w(C(\tau))>4$. Следовательно, $G=P G L(2,25)$.

Пусть $H=P S L\left(2, p^{3}\right), p \in\{3,5,7\}$. Если $G=\operatorname{Aut}(H)$, то из $C(\tau) \geqslant\langle\tau\rangle \times P G L(2, p)$ получаем противоречие с условием $v(G)=4$. Если $G=P G L\left(2, p^{3}\right)$, то $p \neq 7$, так как $\left|S_{2}\left(P G L\left(2,7^{3}\right)\right)\right|=32$. Наконец, если $G=P S L\left(2, p^{3}\right) \lambda\langle\tau\rangle$, то из $C(\tau) \geqslant\langle\tau\rangle \times P S L(2, p)$ следует, что $p=3$.

Предположим теперь, что $H=P S L(2, p)$. Тогда $G=P G L(2, p)$. В этом случае $v(G)=v(H)+1$, т.е. $v(H)=3$ и $p$ удовлетворяет ограничению из пункта 1$)$ теоремы.

Пусть теперь $H$ - элементарная абелева группа порядка $p^{n}$. Так как $C(H)$ разрешим, а $G$ неразрешима, то $n>1$. Кроме того, $n \leqslant 4$.

В дальнейшем мы неоднократно будем использовать лемму 0.5 из [6] и то, что в регулярной группе автоморфизмов любая подгрупша порядка $p q$ циклическая (см. V. 8.12 из [7]).

Если $n=4$, то $p \neq 2$. Так как силовская 2 -подгрупша $S_{2}$ групшы $G$ не является ни циклической ни кватернионной групшой (см. [8]), то $S_{2}$ действует на $H$ нерегулярно. Но тог да $w(C(a))>4$ для некоторого $a \in H \backslash 1$.

Предположим теперь, что $n=2$. Тогда $G / C(H) \leqslant G L(2, p)$ и снова $p \neq 2$. Так как $G / C(H)$ неразрешима, то и $K / C(H)=(G / C(H))^{\prime}$ тоже неразрешима. Но тогда силовская 2 -подгруппа в $K / C(H)$, будучи подгруппой силовской 2 -подгруппы $S L(2, p)$, является либо циклической, либо кватернионной группой. Так как $G$ не может содержать инвариантных 2 -подгрупп порядка 2 или 4 , то $S_{2} \cap C(H)=1$. Но тогда $S_{2}(K)$ либо циклическая, либо кватернионная группа. Из неразрешимости $K$ следует, что $S_{2}(K)$ не может быть циклической. Если же $S_{2}(K)$ кватернионная и $\tau$ - инволюция из $S_{2}(K)$, то в силу [8] $C(\tau) \geqslant K$, что противоречит условию $v(G)=4$.

Итак, $n=3$. Так как $G / H$ неразрешима, то $p>2$. Отметим еще, что все минимальные централизаторы грушы $G$, лежащие в $H$, имеют порядок $p$. В самом деле, если $A-$ минимальный централизатор порядка $p^{2}$ и $g \in G$, то из $A \cap A^{g} \neq 1$ и минимальности $A$ следует, что $A^{g}=A$, т.е. $A \triangleleft G$, что противоречит минимальности $H$. Как и вьше, $S_{2}$ действует на $H$ нерегулярно, т.е. $C\left(a_{1}\right)=H \lambda\left\langle\tau_{1}\right\rangle$ для некоторого $a_{1} \in H$ и инволюции $\tau_{1}$. Так как $H \cap C\left(\tau_{1}\right)$ является минимальным централизатором, то $H \cap C\left(\tau_{1}\right)=\left\langle a_{1}\right\rangle$.

Предположим сначала, что $\tau_{1} \in Z\left(S_{2}\right)$. Тогда $C\left(\tau_{1}\right) \geqslant\left\langle a_{1}\right\rangle \lambda S_{2}$ и $S_{2} /\left\langle\tau_{1}\right\rangle$ циклическая групша. Поэтому $S_{2}=\left\langle\tau_{1}\right\rangle \times\langle f\rangle$ абелева.

Так как $\left\langle a_{1}\right\rangle$ нормализуется $S_{2}$, то $H=\left\langle a_{1}\right\rangle \times A$ и $A$ нормализуется $S_{2}$. Снова $S_{2}$ действует на $A$ нерегулярно и $C\left(a_{2}\right)=H \lambda\left\langle\tau_{2}\right\rangle$ для некоторых $a_{2} \in H$ и инволюции $\tau_{2}$ из $S_{2}$. Из $C\left(\tau_{2}\right) \geqslant\left\langle a_{2}\right\rangle \lambda S_{2}$ получаем, что $S_{2} /\left\langle\tau_{2}\right\rangle$ циклическая, т.е. $S_{2}=\left\langle\tau_{1}\right\rangle \times\left\langle\tau_{2}\right\rangle$. Отметим еще, что если элемент $a_{3}$ выбран так, что $A=\left\langle a_{2}\right\rangle \times\left\langle a_{3}\right\rangle$ и $\left\langle a_{3}\right\rangle$ инвариантна относительно $S_{2}$, то $C\left(a_{3}\right)=H \lambda\left\langle\tau_{1} \tau_{2}\right\rangle$.

Пусть теперь $\tau_{1} \notin Z\left(S_{2}\right)$. Тогда $C\left(\tau_{1}\right) \geqslant\left\langle a_{1}\right\rangle \lambda\left(\left\langle\tau_{1}\right\rangle \times Z\left(S_{2}\right)\right)$ и из цикличности $\operatorname{Aut}\left(\left\langle a_{1}\right\rangle\right)$ следует, что $Z\left(S_{2}\right)$ циклический. Заменяя в рассуждениях предыдущего абзаца группу $S_{2}$ на группу $\left\langle\tau_{1}\right\rangle \times\langle\tau\rangle$, где $\tau$ - инволюция из $Z\left(S_{2}\right)$, мы получим, что $C(a)=H \lambda\langle\tau\rangle$ для некоторого элемента $a \in H$, и остается заменить $\tau_{1}$ на $\tau$. 
Итак, мы показали, что

$$
S_{2}=\left\langle\tau_{1}\right\rangle \times\left\langle\tau_{2}\right\rangle, \quad H=\left\langle a_{1}\right\rangle \times\left\langle a_{2}\right\rangle \times\left\langle a_{3}\right\rangle
$$

$S_{2}$ нормализует $\left\langle a_{i}\right\rangle$ и $C\left(a_{i}\right)=H \lambda\left\langle\tau_{i}\right\rangle, i=1,2,3$, где $\tau_{3}=\tau_{1} \tau_{2}$.

Число элементов, сопряженных с $a_{1}$, равно $|G| /\left|C\left(a_{1}\right)\right|=|G| / 2 p^{3}$ и не превосходит $p^{3}-1$. Поэтому $|G| \leqslant 2 p^{3}\left(p^{3}-1\right)$. Отсюда $|G / H| \leqslant 2\left(p^{3}-1\right)$. Так как любая группа порядка менњше 60 разрешима, то $p>3$.

Пусть $x$ - элемент взаимно простого с числом $p$ порядка, $g \in G$ и $[x, g] \in H$. Тогда $x^{g} \in\langle x\rangle H$. Так как в $\langle x\rangle H$ все холловы $p^{\prime}$-подгрупшы сопряжены, то $\left\langle x^{g}\right\rangle=\left\langle x^{a}\right\rangle$ для некоторого $a \in H$. Отсюда $x^{g}=\left(x^{\alpha}\right)^{a}$ и из $[x, g]=x^{-1} a^{-1} x^{\alpha} a=x^{\alpha-1}\left[x^{\alpha}, a\right] \in H$ следует, что $x^{\alpha-1} \in H$, т.е. $\alpha=1$. Но тогда $x^{g}=x^{a}$ и $g \in C(x) H$, т.е. $C_{G / H}(x H)=C(x) H / H$. Это означает, в частности, что если $K$ - такая $p^{\prime}$-подгруппа группы $G$, что для любого элемента $h$ простого порядка из $K$ вьполняется включение $C(h) \leqslant K \cdot H$, то $(K \cdot H) / H$ сильно изолирована в $G / H$.

Если для любой инволюции $\tau_{i} \in S_{2}$ вьполняется равенство $C\left(\tau_{i}\right)=\left\langle a_{i}\right\rangle \lambda S_{2}$, то $\left(S_{2} H\right) / H$ сильно изолирована в $G / H$.

Пусть $C(\tau)>\langle a\rangle \lambda S_{2}$ для некоторой инволюции $\tau \in S_{2}$. Тогда $C(\tau)=\langle a\rangle \lambda K$ и из цикличности $K /\langle\tau\rangle \leqslant \operatorname{Aut}(\langle a\rangle)$ следует, что $K$ абелева, т.е. $K=S_{2} \times\langle b\rangle,|b|=q-$ простое число. Если $C(b)=K$, то подгруппа $(K \cdot H) / H$ сильно изолирована в $G / H$.

Предположим теперь, что $C(b)>K$. Если $C(b) \cap H \neq 1$, то $C(b) \cap H$ нормализуется $S_{2}$. Но тогда $C(C(b) \cap H) \ni \tau_{i}$ для некоторого $i=1,2,3$ и $C\left(a_{i}\right) \geqslant\left\langle H, \tau_{i}, b\right\rangle$, что невозможно. Поэтому $C(b) \cap H=1$. Следовательно, $C(\tau) \cap C(b)=K$. Так как $|C(b) /\langle b\rangle|=4 r$ для некоторого простого числа $r$, то $S_{2}$ инвариантна в $C(b)$ и $r=3$.

Если $C(b)=S_{2} \lambda\langle x\rangle, x^{9}=1, x^{3}=b$, то элементы простого порядка из $C(b)$ исчерпываются инволюциями из $S_{2}$ и элементами, сопряженными с $b$ и $b^{2}$, т.е. $C(b) H / H$ сильно изолирована в $G / H$.

Пусть $C(b)=\left(S_{2} \times\langle b\rangle\right) \lambda\langle x\rangle, x^{3}=1$. Тогда можно считать, что $\tau_{1}^{x}=\tau_{2}, \tau_{2}^{x}=\tau_{1} \tau_{2}$. Из $C\left(\tau_{i}^{x}\right)=C\left(\tau_{i}\right)^{x}$ следует, что $a_{1}^{x}=a_{2}^{t_{1}},\left(a_{2}^{t_{1}}\right)^{x}=a_{3}^{t_{2}}$ и $\left(a_{3}^{t_{2}}\right)^{x}=a_{1}$ для некоторых чисел $t_{1}, t_{2}$. Но тог да $a=a_{1} a_{2}^{t_{1}} a_{3}^{t_{2}} \in C(x)$, т.е. $C(x) \geqslant\langle a\rangle \lambda(\langle b\rangle \times\langle x\rangle)$. Из доказанного, в частности, следует, что для любого элемента $x$ порядка 3 из $N\left(S_{2}\right)$ вьполняется $C(x) \cap H \neq 1$.

Положим $C(x)=\langle a\rangle \lambda R, b \in R$. Так как $R /\langle x\rangle \leqslant \operatorname{Aut}(\langle a\rangle)$, то $R$ абелева. Но тогда $R \leqslant C(x) \cap C(b)=\langle b\rangle \times\langle x\rangle$, т.е. $C(x)=\langle a\rangle \lambda(\langle b\rangle \times\langle x\rangle)$. А это, как нетрудно видеть, означает, что $C(b) H / H$ сильно изолирована в $G / H$.

Итак, мы показали, что в фактор-групше $G / H$ есть сильно изолированная подгруппа четного порядка. По [9] $G / H$ изоморфна либо $P S L\left(2,2^{n}\right)$, либо $S z\left(2^{2 n+1}\right)$ для некоторого числа $n$. Так как $\left|S_{2}\right|=4$, то $G / H \cong P S L(2,4) \cong P S L(2,5)$.

Предположим сначала, что $p>5$. Тогда $G=H 入 K$, где $K \cong P S L(2,5)$. Группа $P S L(2,5)$ содержит 15 подгрупп порядка 2,10 подгрупп порядка 3 и 6 подгрупп порядка 5. Из проведенных вьше рассуждений следует, что для любого элемента $x \in K$ порядка 2 или 3 выполняется равенство $|H \cap C(x)|=p$. Заметим, что для $a \in H$ и $x \in K$ из $C\left(x^{a}\right)=C(x)^{a}$ следует $C(x) \cap H=C\left(x^{a}\right) \cap H$. Поэтому для $g=h a, h \in K$, имеем $C\left(x^{g}\right) \cap H=C\left(x^{h}\right) \cap H$.

Если для элемента $y$ порядка 5 вьполняется равенство $|H \cap C(y)|=1$, то среди неединичных элементов $H$ содержится $25(p-1)$ элементов со свойством $C(x)>H$ 
и $p^{3}-1-25(p-1)$ элементов $x$ со свойством $C(x)=H$. Так как $|G / H|=60$, то $p^{3}-1=25(p-1)+60 t$ для некоторого числа $t>0$. Но тогда $p^{3}-1$, а следовательно, и $p-1$ делится на 10 .

Если же для элемента $y$ порядка 5 выполняется равенство $|H \cap C(y)|=p$, то аналогично получим $p^{3}-1=31(p-1)+60 t, t>0$. Из $(p-1)\left(p^{2}+p-30\right)=60 t$ следует, что либо $p-1$, либо $p+1$ делится на 10 .

В случае $p=5$, как нетрудно видеть, для любого $x \in H \backslash 1 C(x) / H$ является групой простого порядка, и если $C(x)=C(y)$ для $x, y \in H$, то $\langle x\rangle=\langle y\rangle$, т.е. $G$-группа типа 3$)$, и теорема доказана.

2. Разрешимые группы. Рассмотрим теперь случай разрешимой группы $G$. В этом случае, если $F=F(G)$ - подгруппа Фиттинга группы $G$, то $C(F) \leqslant F$ (см. [7, III.4.2]). Поэтому $w(F) \leqslant 4$.

Число неизоморфных групп в этом случае весьма велико. Поэтому мы не будем доводить до полного описания, предполагая, что групы, заданные данными отношениями, существуют. Так, например, если сказано, что $\langle a\rangle \lambda\langle b\rangle$ - группа Фробениуса, то это предполагает, что $|b|$ делит $|a|-1$ и $\langle b\rangle$ действует на $\langle a\rangle$ регулярно. Все числа $p, q, p_{i}$, $q_{i}, r$ и т.д. являются простыми числами.

Теорема 2.1. Пусть $G$ - конечная разрешимая группа без иентра, $v(G)=4$ u $G$ не является $\mathfrak{M - z р у п п о и ̆ . ~ П о д г р у п п а ~} F$ абелева тогда и только тогда, когда выполняется один из следующих случаев:

1. $G=F \lambda K-$ группа Фробениуса, $F$ - абелева типа $(p, p),(p, p, p),(p, p, q, q)$, $\left(p^{2}, p^{2}\right), K$ - одна из следующих групп: $Q_{8} ; Q_{16} ; Q_{8} \times\langle a\rangle$ или $\langle a\rangle \lambda Q_{8}$, $|a|=q \neq 2 ; Q_{8} \lambda\langle a\rangle, a^{3}=1 ;\langle a\rangle \lambda\langle b\rangle$, при этом либо $|a|=p_{1},|b|=q_{1}^{2} s$, $s=1$ или $s$ - простое число, $[a, b] \neq 1,\left[a, b_{1}^{q}\right]=1$, либо $|a|=p_{1} p_{2},|b|=q_{1}^{2}$, $[a, b] \neq 1,\left[a, b_{1}^{q}\right]=1,(|a|,|b|)=1 u \max \{w(F), w(K)\}=4$;

2. $G=G_{1} \times G_{2}, G_{1} u G_{2}$ - группы Фробениуса порядков $p_{1} p_{2}$ и $q_{1} q_{2} q_{3}$ соответственно;

3. $G=\left(\left\langle b_{1}\right\rangle \times\left\langle b_{2}\right\rangle\right) \lambda\langle c\rangle, b_{1}^{p}=b_{2}^{q}=1, w(|c|)=3, \max _{b \in F \backslash 1} w(C(b) \cap\langle c\rangle)=1$ uлu $2, C\left(b_{1}\right) \cap C\left(b_{2}\right)=F$;

4. $G=F \lambda\langle c\rangle, w(F)=3, w(|c|)=2$ uлu $3, \max _{b \in F \backslash 1} w(C(b) \cap\langle c\rangle)=1, C\left(b_{1}\right) \cap$ $C\left(b_{2}\right)=F$ для некоторых $b_{1}, b_{2} \in F$;

5. $G=\left(\left\langle b_{1}\right\rangle \times\left\langle b_{2}\right\rangle\right) \lambda\left(\left\langle a_{1}\right\rangle \times\left\langle a_{2}\right\rangle\right), b_{1}^{p}=b_{2}^{p}=a_{1}^{q}=a_{2}^{q r}, q \neq r,\left[a_{1}, b_{1}\right]=\left[a_{2}^{r}, b_{2}\right]=1$, $\left[a_{2}, b_{2}\right] \neq 1,\left\langle b_{1}\right\rangle \lambda\left\langle a_{2}\right\rangle$ u $\left\langle b_{2}\right\rangle \lambda\left\langle a_{1} a_{2}^{q}\right\rangle-$ zруппь Фробениуса;

6. $G=\left(\left\langle b_{1}\right\rangle \times\left\langle b_{2}\right\rangle\right) \lambda\left(\left(\left\langle a_{1}\right\rangle \times\left\langle a_{2}\right\rangle\right) \lambda\langle\tau\rangle\right), b_{1}^{p}=b_{2}^{p}=\tau^{2}=a_{1}^{q}=a_{2}^{q r}=1, a_{2}^{\tau}=a_{2}$, $b_{1}^{\tau}=b_{2}, a_{1}^{\tau}=a_{1}^{-1} a_{2}^{r},\left[a_{1}, b_{1}\right]=1,\left\langle a_{1}\right\rangle$ на $\left\langle b_{2}\right\rangle u\left\langle a_{2}\right\rangle$ на $\left\langle b_{1}\right\rangle$ и $\left\langle b_{2}\right\rangle$ действуют регулярно;

7. $G=\left(\left\langle b_{1}\right\rangle \times\left\langle b_{2}\right\rangle\right) \lambda(\langle a\rangle \lambda\langle\tau\rangle), b_{1}^{p}=b_{2}^{p}=\tau^{2}=1, b_{1}^{\tau}=b_{2}, a^{\tau}=a^{p},\langle a\rangle$ действуетна $\left(\left\langle b_{1}\right\rangle \times\left\langle b_{2}\right\rangle\right)$ неприводимо, $w(|a|)=4$, если $(|a|, p-1)=1, u$ $w(|a|)=3$ в противном случае;

8. $F=\left(\left\langle b_{1}\right\rangle \times\left\langle b_{2}\right\rangle\right), b_{1}^{3}=b_{2}^{3}=1, G / F \cong S L(2,3)$ и действует на $F$ естественным образом;

9. $G=\left(\left\langle b_{1}\right\rangle \times\left\langle b_{2}\right\rangle\right) \lambda(\langle x\rangle \lambda\langle y\rangle), b_{1}^{p}=b_{2}^{p}=x^{q^{2}}=1, w(|y|)=2$ uлu $3,\left[x^{q}, b_{i}\right]=1$, $i=1,2,\langle x\rangle$ действует на $\left\langle b_{1}\right\rangle \times\left\langle b_{2}\right\rangle$ неприводимо, $\langle x\rangle \lambda\langle y\rangle-$ группа Фробениуса, $\max _{i=1,2} w\left(C\left(b_{i}\right) \cap\langle y\rangle\right)=1, C\left(b_{1}\right) \cap C\left(b_{2}\right)=\left\langle b_{1}\right\rangle \times\left\langle b_{2}\right\rangle \times\left\langle x^{q}\right\rangle$; 
10. $G=\left(\left\langle b_{1}\right\rangle \times\left\langle b_{2}\right\rangle \times\langle c\rangle\right) \lambda(\langle x\rangle \lambda\langle y\rangle), b_{1}^{p}=b_{2}^{p}=c^{q}=x^{r}=1,|y|=t^{2} s, t-$ простое число, s либо равно 1 , либо простое число, $c^{x}=c,\langle c\rangle \lambda\langle y\rangle u$ $\left(\left\langle b_{1}\right\rangle \times\left\langle b_{2}\right\rangle\right) \lambda(\langle x\rangle \lambda\langle y\rangle)$ группь Фробениуса, $C(x) \cap\langle y\rangle$ равно либо $\left\langle y^{t}\right\rangle$, либо $\left\langle y^{t^{2}}\right\rangle$, причем последнее возможно только при $s=t$;

11. $G=\left(\left\langle b_{1}\right\rangle \times\left\langle b_{2}\right\rangle \times\langle c\rangle\right) \lambda(\langle a\rangle \lambda\langle\tau\rangle), b_{1}^{p}=b_{2}^{p}=c^{q}=x^{r}=\tau^{2}=1, r$ - нечетный простой делитель числа $p+1,\langle a\rangle$ действует на $\left\langle b_{1}\right\rangle \times\left\langle b_{2}\right\rangle$ неприводимо, $c^{a}=c, c^{\tau}=c^{-1}, b_{1}^{\tau}=b_{2}$

12. $G=\left(\left\langle b_{1}\right\rangle \times\left\langle b_{2}\right\rangle \times\left\langle b_{3}\right\rangle\right) \lambda(\langle x\rangle \lambda\langle y\rangle), b_{1}^{p}=b_{2}^{p}=b_{3}^{p}=y^{3}=1,\left[b_{1}, y\right]=1$, $\left(\left\langle b_{1}\right\rangle \times\left\langle b_{2}\right\rangle \times\left\langle b_{3}\right\rangle\right) \lambda\langle x\rangle u\left(\left\langle b_{2}\right\rangle \times\left\langle b_{3}\right\rangle\right) \lambda\langle y\rangle$ группь Фробениуса, $|x|$ делит $p^{3}-1$ и не делит $p-1$; если $(|x|, p-1)=1$, то $w(|x|) \leqslant 4$, иначе $w(|x|) \leqslant 3$, $x^{y}=x^{p}$.

ДоКАЗАТЕЛЬСТво. Пусть $G$ - группа из условия теоремы. Предположим сначала, что $G=F \lambda K$ - группа Фробениуса. Если $K$ абелева, то по теореме 5.12 из [3] $G$ является $\mathfrak{M}$-группй. Поэтому $K$ неабелева. Из описания разрешимых групп регулярных автоморфизмов (см., например, $[10], \S 6)$ и условия $v(G)=4$ получаем группы из пункта 1 теоремы.

Пусть теперь $G$ не является группой $ф$ робениуса. Тогда найдутся неединичные элементы $b \in F$ и $g \in G \backslash F$ со свойством $[b, g]=1$. Поэтому $w(F) \leqslant 3$. Так как $F=C(F), Z(G)=1$ и $G / F$ действует на $F$ нерегулярно, то $w(F) \geqslant 2$. Рассмотрим случай $w(F)=2$. Если $F=\langle b\rangle$ - циклическая, то $G=\langle b\rangle\langle x\rangle$ для некоторого элемента $x \in G$, и из $Z(G)=1$ получаем $G=\langle b\rangle \lambda\langle x\rangle$. Пусть $|b|=p^{2}$. Так как $\langle x\rangle$ действует на $\langle b\rangle$ нерегулярно, то $(|x|, p) \neq 1$. Но тогда $S_{p}(G) \triangleleft G$ и $S_{p}(G)>F$, что невозможно. Поэтому $F=\langle a\rangle \times\langle b\rangle, a^{p}=b^{q}=1$. Если $p \neq q$, то снова $G=\langle a b\rangle \lambda\langle x\rangle$ и мы получим группу типа 2 или 3 .

Пусть теперь $F=\left\langle b_{1}\right\rangle \times\left\langle b_{2}\right\rangle$ - элементарная абелева групша порядка $p^{2}$. Тогда $G / F \leqslant \operatorname{Aut}(F)=G L(2, p)$. Из $v(G) \neq 3$ следует, что $p>2$. Так как группа $G$ разрешима, то $G / F$ содержится в максимальной разрешимой подгруппе группы $G L(2, p)$.

Максимальные разрешимые подгруппы группы $G L(2, p)$ известны $([11, \S 21])$. В частности, если $p>3$ и $H$ - максимальная разрешимая подгруппа из $G L(2, p)$, то при соответствующем выборе базиса вьполняется один из следующих случаев:

- если $H$ приводима, то она является треугольной группой (в частности, силовская $p$-подгруппа из $H$ инвариантна в $H$ );

- если $H$ неприводима и импримитивна, то

$$
H=\left(\left\langle a_{1}\right\rangle \times\left\langle a_{2}\right\rangle\right) \lambda\langle\tau\rangle, \quad a_{1}^{p-1}=a_{2}^{p-1}=\tau^{2}=1, \quad a_{1}^{\tau}=a_{2}
$$

- если $H$ неприводима и примитивна, то либо

$$
H=\langle a\rangle \lambda\langle\tau\rangle, \quad a^{p^{2}-1}=\tau^{2}=1, \quad a^{\tau}=a^{p},
$$

либо

$$
H / Z \cong\left(\left\langle a_{1}\right\rangle \times\left\langle a_{2}\right\rangle\right) \lambda G L(2,2), \quad Z=Z(G L(2, p)), \quad a_{1}^{2}=a_{2}^{2}=1,
$$

и $G L(2,2)$ действует на $\left\langle a_{1}\right\rangle \times\left\langle a_{2}\right\rangle$ естественным образом. 
Предположим сначала, что $G / F$ приводима. Так как силовская $p$-подгрупша в $G / F$ инвариантна, то $(|G / F|, p)=1$, т.е. $G=F \lambda K$, где $K$ изоморфна подгрупше грушы диагональных матриц. Если $K$ циклическая, то снова получим группу типа 2 или 3 . Если же $K$ не циклическая, то из цикличности фактор-групш $G / C\left(b_{1}\right)$ и $G / C\left(b_{2}\right)$ следует, что $G$ группа типа 2 или типа 5 .

Если $G / F$ неприводима, то $G=F \lambda K$, где $K$ - неприводимая разрешимая подгруппа из $G L(2, p)$. Если $K$ импримитивна, то из неприводимости ее и условия $v(G)=4$ следует, что $G$ - групша типа 6 .

Пусть теперь $K$ примитивна и $K \leqslant\langle a\rangle \lambda\langle\tau\rangle, a^{p^{2}-1}=\tau^{2}=1$. Так как $\langle a\rangle$ действует на $F$ регулярно, то можно считать, что $\tau \in K$ и мы получаем группу типа 7 .

Наконец, пусть $G=F \lambda K$ и $K$ содержится в подгрупше $H$ из $G L(2, p)$ со свойством $H / Z=\left(\left\langle\bar{a}_{1}\right\rangle \times\left\langle\bar{a}_{2}\right\rangle\right) \lambda G L(2,2)$. Так как $K$ примитивна, то по лемме 21.5 из [11] $K Z / Z$ содержит элемент порядка 3 . Но тогда

$$
K Z / Z=\left(\left\langle\bar{a}_{1}\right\rangle \times\left\langle\bar{a}_{2}\right\rangle\right) \lambda\langle\bar{c}\rangle, \quad \bar{c}^{3}=1, \quad \bar{a}_{1}^{\bar{c}}=\bar{a}_{2}, \quad \bar{a}_{2}^{\bar{c}}=\bar{a}_{1} \bar{a}_{2} .
$$

Так как $\left[a_{1}, a_{2}\right]$ - элемент порядка 2 из $Z$, то $\left|\left\langle a_{1}, a_{2}\right\rangle\right| \geqslant 8$. Если $\left|\left\langle a_{1}, a_{2}\right\rangle\right|>8$, то $w(K \cap Z)>1$. Но тогда для $x \in(K \cap Z) \backslash 1$ будем иметь $w(C(x))>4$, что невозможно. Поэтому $\left\langle a_{1}, a_{2}\right\rangle$ может быть только группой кватернионов порядка 8 .

Итак, $G=F \lambda\left(\left\langle a_{1}, a_{2}\right\rangle \lambda\langle c\rangle\right)$. Так как $a_{1}^{2} \in Z$, то $a_{1}^{2}$, а следовательно, и $\left\langle a_{1}, a_{2}\right\rangle$ действуют на $F$ регулярно. Поэтому $C(c) \cap F \neq 1$. Положим $\left[b_{1}, c\right]=1$. Так как $a_{1}^{2} \in N(\langle c\rangle)$, то $a_{1}^{2} \in N\left(\left\langle b_{1}\right\rangle\right)$. Пусть $F=\left\langle b_{1}\right\rangle \times\left\langle b_{2}\right\rangle$, где $\left\langle b_{2}\right\rangle=\left[F, a_{1}^{2} c\right]$. Тогда $b_{2}^{c}=b_{2}^{\alpha}$, $\alpha^{3}=1, b_{i}^{a_{1}^{2}}=b_{i}^{-1}$. То есть мы можем отождествить $a_{1}^{2}$ и $c$ с матрицами $\left(\begin{array}{cc}-1 & 0 \\ 0 & -1\end{array}\right)$ и $\left(\begin{array}{ll}1 & 0 \\ 0 & \alpha\end{array}\right)$ соответственно. Но тогда если $a_{1}=\left(\begin{array}{cc}x & y \\ z & t\end{array}\right)$, то $a_{2}=a_{1}^{c}=\left(\begin{array}{cc}x & \alpha y \\ \alpha z & t\end{array}\right)$, что невозможно, так как в этом случае $\left[a_{1}, a_{2}\right] \neq a_{1}^{2}$.

Если же $p=3$, то $G / F$ является подгруппой групшы $G L(2,3)$. Из $v(G)=4$ следует, что $G / F<G L(2,3)$. Если $(|G / F|, p)=1$, то мы получим группу типа 7 . А если $G / F$ содержит 3 -элемент, то группу типа 8.

Предположим теперь, что $w(F)=3$. Если $G / F$ циклическая, то снова $G=F \lambda\langle x\rangle$ и получим групп типа 4 . Пусть $G / F$ - нециклическая группа. Тогда и группа $F$ тоже нециклическая. Предположим, что

$$
F=\left\langle b_{1}\right\rangle \times\left\langle b_{2}\right\rangle \times\langle c\rangle, \quad b_{1}^{p}=b_{2}^{p}=c^{q}=1,
$$

где $p$ и $q$ различные простые числа. Так как $G / F$ нециклическая, то $C(c)>F$. Положим $C(c)=F\langle x\rangle$. Тогда $x^{r} \in F$ для некоторого простого числа $r$ и $G / C(c)$ циклическая группа.

Если $\left\langle b_{1}\right\rangle \triangleleft G$, то $\left\langle b_{1} c\right\rangle \triangleleft G$ и $G / C\left(b_{1} c\right)$ циклическая. Но тогда из $C\left(b_{1} c\right) \leqslant C(c)$ следует $C\left(b_{1}\right)=C(c)$. Если $r \neq p$, то можно считать, что $\left\langle b_{2}\right\rangle \triangleleft G$. Но тогда снова $C\left(b_{2}\right)=C(c)$ и $C(F)=C(c)>F$, что невозможно. Если же $r=p$, то $C\left(b_{1}\right)$ - нильпотентная нормальная подгрупша, что тоже невозможно. Поэтому $\left\langle b_{1}\right\rangle \times\left\langle b_{2}\right\rangle-$ минимальный нормальньй делитель групшы $G$ и $C(b c)=F$ для любого неединичного $p$-элемента $b$ из $F$.

Предположим сначала, что $G / F$ абелева, т.е. $G / F=\langle\bar{x}\rangle \times\langle\bar{y}\rangle$. Из нецикличности $G / F$ следует, что $|\bar{y}|$ делится на $|r|$. Из $v(G)=4$ следует, что $p \neq r \neq q$. Так как для любого неединичного $p$-элемента $b$ из $F C(b) \triangleleft G$, то и $C^{2}(b) \Delta G$. По уже доказанному 
$C^{2}(b) \geqslant\left\langle b_{1}\right\rangle \times\left\langle b_{2}\right\rangle$, т.е. $C(b)=C\left(\left\langle b_{1}\right\rangle \times\left\langle b_{2}\right\rangle\right)$. Пусть $\bar{y}_{0}$ - элемент порядка $r$ из $\langle\bar{y}\rangle$. Так как $\langle\bar{x}\rangle \times\left\langle\bar{y}_{0}\right\rangle-$ нециклическая абелева группа, то она действует на $\left\langle b_{1}\right\rangle \times\left\langle b_{2}\right\rangle$ не регулярно, т.е. можно считать, что $y_{0} \in C\left(b_{1}\right)=C\left(\left\langle b_{1}, b_{2}\right\rangle\right)$. Из $C\left(y_{0}\right) \geqslant\left\langle x, y, b_{1}, b_{2}\right\rangle$ следует, что $G$ - групша типа 2.

Пусть теперь $G / F=\langle\bar{x}\rangle \lambda\langle\bar{y}\rangle$ неабелева. Так как $\left\langle b_{1}\right\rangle \times\left\langle b_{2}\right\rangle$ минимальньй нормальный делитель группы $G$, то $G / C\left(\left\langle b_{1}, b_{2}\right\rangle\right)$ действует на $\left\langle b_{1}\right\rangle \times\left\langle b_{2}\right\rangle$ неприводимо. Предположим сначала, что $p>3$. Из приведенного выше описания максимальных неприводимых разрешимых подгрупп групш $G L(2, p)$ следует, что в этом случае $\left(\left|G / C\left(\left\langle b_{1}, b_{2}\right\rangle\right)\right|, p\right)=1$. Поэтому либо $\left\langle b_{1}\right\rangle \times\left\langle b_{2}\right\rangle$ - силовская $p$-подгруппа группы $G$, либо $C\left(\left\langle b_{1}\right\rangle \times\left\langle b_{2}\right\rangle\right)=F\langle t\rangle, t^{p} \in F$. Рассмотрим второй случай. Мы можем считать, что $t \in\langle y\rangle$.

Пусть $H=\left\langle C\left(b_{1}\right), C(c)\right\rangle$. Тогда $H / F=\langle\bar{x}\rangle \times\langle\bar{t}\rangle-$ абелева групша. Так как $r \neq p$, то $H / F$ циклическая. Из $Z(H)=1$ следует, что $H=F \lambda\langle x t\rangle$, т.е. $x \in C(t)$. Но тогда, рассматривая $C(t)$, как и выше получим $y=t$, что противоречит неабелевости $G / F$.

Итак, мы можем считать, что $\left\langle b_{1}\right\rangle \times\left\langle b_{2}\right\rangle-$ силовская $p$-подгруппа групшы $G$. Но тогда

$$
G=\left(\left\langle b_{1}\right\rangle \times\left\langle b_{2}\right\rangle\right) \lambda(\langle c, x\rangle \lambda\langle y\rangle) .
$$

Предположим, что $\langle c, x\rangle=\langle x\rangle$ - циклическая группа порядка $q^{2}$. Тогда $\langle x\rangle \lambda\langle y\rangle-$ группа Фробениуса. Так как подгруппа $\left(\left\langle b_{1}\right\rangle \times\left\langle b_{2}\right\rangle\right) \lambda\langle x\rangle$ не нильпотентна, то $\langle y\rangle$ действует на ней нерегулярно. Поэтому можно считать, что $C\left(b_{1}\right) \cap\langle y\rangle=\left\langle y_{0}\right\rangle \neq 1$. Отсюда $w(|y|) \leqslant 3$.

Если $C\left(b_{2}\right)=C\left(b_{1}\right)$, то подгруппа

$$
\langle c\rangle \lambda\left\langle y_{0}\right\rangle=C\left(\left\langle b_{1}, b_{2}\right\rangle\right) \cap(\langle x\rangle \lambda\langle y\rangle)
$$

нормальна в $\langle x\rangle \lambda\langle y\rangle$, т.е. $\left[x, y_{0}\right] \leqslant\langle c\rangle$. Из $\langle x\rangle=\left[\langle x\rangle,\left\langle y_{0}\right\rangle\right] \times C_{\langle x\rangle}\left(y_{0}\right)$ следует, что $[x, y]=1$, что невозможно. Поэтому $C(b) \neq C\left(b_{1}\right)$ для любого $b \in\left(\left\langle b_{1}\right\rangle \times\left\langle b_{2}\right\rangle\right) \backslash\left\langle b_{1}\right\rangle$. Если $C\left(b_{2}\right) \cap\langle y\rangle=\left\langle y_{1}\right\rangle$, то $\left\langle y_{0}\right\rangle \cap\left\langle y_{1}\right\rangle=1$ и мы получаем группу типа 9 .

Предположим теперь, что $\langle c, x\rangle=\langle c\rangle \times\langle x\rangle$. Как и в предыдущем случае, $C\left(b_{1}\right) \neq$ $C\left(b_{2}\right)$. Если $\langle x\rangle \lambda\langle y\rangle$ действует на $\left\langle b_{1}\right\rangle \times\left\langle b_{2}\right\rangle$ регулярно, то получим группу типа 10 . Если же $\langle x\rangle \lambda\langle y\rangle$ действует на $\left\langle b_{1}\right\rangle \times\left\langle b_{2}\right\rangle$ нерегулярно, то, применяя к подгруппе $H=$ $\left(\left\langle b_{1}\right\rangle \times\left\langle b_{2}\right\rangle\right) \lambda(\langle x\rangle \lambda\langle y\rangle)$ проведенные выше рассуждения о неприводимых разрешимых подгруппах из $G L(2, p)$, получим, что $H$ может быть только группой типа 7 при $x=a$, т.е. $G$ - группа типа 11 .

Предположим теперь, что $p \leqslant 3$. Если $(|G / F|, p)=1$, то новых групп не появится. Пусть $(|G / F|, p) \neq 1$. Если при этом $r \neq p$, то $r-1$ делится на $p$, а

$$
|G L(2, p)|=p(p-1)^{2}(p+1)
$$

делится на $r$. Это возможно только при $p=2$ и $r=3$. Но тогда если $C\left(\left\langle b_{1}, b_{2}\right\rangle\right)=F$, то $G$ - группа типа 11 . Если же $C\left(\left\langle b_{1}, b_{2}\right\rangle\right)>F$, то фактор-группа $G / F$ будет абелевой. В случае $r=p$ подгруппа $C(c)$ является нильпотентной нормальной подгруппой группы $G$, что невозможно.

Пусть теперь $F=\left\langle b_{1}\right\rangle \times\left\langle b_{2}\right\rangle$, где $b_{1}^{p^{2}}=b_{2}^{p}=1$. Так как $\left\langle b_{1}^{p}\right\rangle \triangleleft G$, то и $C\left(b_{1}^{p}\right) \triangleleft G$. Поэтому $F$ - силовская $p$-подгруппа групп $G$ и $G=F \lambda K$. Если группа $K$ циклическая, то $G$ - группа типа 2 или 4 . 
Пусть $K$ нециклическая. Тогда $C\left(b_{1}^{p}\right)>F$. Так как для любого элемента $t \in K \backslash 1$ выполняется равенство $F=[F, t] \times C_{F}(t)$, то $C_{F}(t)$ либо равен 1 , либо является максимальной циклической подгруппой из $F$. Поэтому $C\left(b_{1}^{p}\right)=C\left(b_{1}\right)$. Из цикличности фактор-группы $G / C\left(b_{1}^{p}\right)$ следует, что $G=F \lambda(\langle x\rangle \lambda\langle y\rangle), x^{q}=1,\left[b_{1}, x\right]=1$. Так как $C\left(b_{1}\right) \triangleleft G$, то $\left\langle b_{1}\right\rangle=C^{2}\left(b_{1}\right) \triangleleft G$ и, следовательно, можно считать, что $\left\langle b_{2}\right\rangle \triangleleft G$, т.е. $G / C\left(b_{2}\right)$ циклическая. Поэтому $K=\langle x\rangle \times\langle y\rangle$ - нециклическая абелева группа. Из $w(C(x)) \leqslant 4$ получаем $w(K)=2$. Но тогда $G-$ группа типа 2 .

Наконец, рассмотрим случай, когда $F=\left\langle b_{1}\right\rangle \times\left\langle b_{2}\right\rangle \times\left\langle b_{3}\right\rangle$ - элементарная абелева группа порядка $p^{3}$. Тогда $G / F$ изоморфна разрешимой подгрупе из $G L(3, p)$. Если $G / F$ циклическая, то $G$ - группа типа 2 или 4 . Если $G / F$ приводима, то, повторяя рассуждения, проведенные в случае $|F|=p^{2} q$, получим либо группу типа 2 , либо группу типа 11 при $p=q$. Поэтому будем считать, что $G / F$ неприводима.

Предположим сначала, что $p>3$. Строение максимальных неприводимых разрешимых подгрупп групшы $G L(3, p)$ известно (теорема 21.6 из [11]). В силу этой теоремы $(|G / F|, p)=1$ и $G=F \lambda K$. Если $K$ импримитивна, то $K$ является подгруппой сплетения циклической группы порядка $(p-1)$ и симметрической группы $S_{3}$. Но тогда, как несложно проверить, $v(G)>4$. Поэтому группа $K$ примитивна.

Пусть $K \leqslant H=\langle a\rangle \lambda\langle y\rangle,|a|=p^{3}-1,|y|=3$. В этом случае если $F$ отождествить с $G F\left(p^{3}\right)$, то $\langle a\rangle$ действует на $F$ как умножение на элементы из $G F\left(p^{3}\right)^{*}$, а $y$ как автоморфизм поля. Так как $v(G)=4$, то $G$ группа типа 12 .

Если же $K$ содержится в такой подгрупше $H$ групшы $G L(3, p)$, что $H / Z=\left(\left\langle\bar{a}_{1}\right\rangle \times\left\langle\bar{a}_{2}\right\rangle\right) \lambda$ $S L(2,3), Z=Z(G L(3, p)), \bar{a}_{1}^{3}=\bar{a}_{2}^{3}=1$ и $S L(2,3)$ действует на $\left\langle\bar{a}_{1}\right\rangle \times\left\langle\bar{a}_{2}\right\rangle$ естественным образом (такое возможно только в случае $p \equiv 1(3)$ ), то снова получим $v(G)>4$.

В случае $p=2$ максимальные неприводимые разрешимые подгруппы группы $G L(3,2)$ исчерпываются неабелевыми групшами порядка 21 , т.е. $G$ и в этом случае будет группой типа 12. Наконец, если $p=3$, то снова либо $K$ импримитивна и $v(G)>4$, либо $K$ примитивна и $G$ - группа типа 12 .

В дальнейшем через $\wp(n)$ условимся обозначать показатель числа $n$ по простому модулю $p$.

Теорема 2.2. Пусть $G$ - конечная разрешимая группа без центра и $v(G)=4$. Подгруппа $F$ неабелева тогда и только тогда, когда выполняется один из следующих случаев:

1. $G=F \lambda\langle c\rangle-$ группа Фробениуса, $F$ - неабелева группа порядка $p^{3}, p^{4}$ или $p^{3} q, p$ и $q$ - простые числа, $w(|c|)=4$ в первом случае $u w(|c|) \leqslant 4$ в остальных случаях;

2. $G=(((\langle z\rangle \times\langle a\rangle) \lambda\langle b\rangle) \times\langle x\rangle) \lambda\langle c\rangle, z^{p}=a^{p}=b^{p}=x^{q}=1,[a, b]=z,\langle z\rangle \lambda\langle c\rangle$ $u\langle x\rangle \lambda\langle c\rangle$ - группь Фробениуса, $w(|c|) \leqslant 3, a^{c}=a^{\alpha}, b^{c}=b^{\beta}, z^{c}=z^{\alpha \beta}$, $\wp(\alpha \beta)=|c|$ и либо $\wp(\beta)=|c|, \wp(\alpha)=|c| / r$, либо $\wp(\beta)=|c| / r_{1}, \wp(\alpha)=|c| / r_{2}$, $r, r_{1}, r_{2}$ - простые делители $|c|, r_{1} \neq r_{2}$

3. $G=((\langle b\rangle \lambda\langle a\rangle) \times\langle x\rangle) \lambda\langle c\rangle, b^{p^{2}}=a^{p}=x^{q}=1,[a, b]=b^{p},\langle b\rangle \lambda\langle c\rangle u\langle x\rangle \lambda\langle c\rangle-$ группь Фробениуса, $[a, c]=1, w(|c|)=1$;

4. $G=(\langle a\rangle \lambda\langle b\rangle) \lambda\langle c\rangle, a^{p^{3}}=b^{p}=1,[a, b]=a^{p^{2}}, w(|c|)=1,\langle a\rangle \lambda\langle c\rangle-$ zpynna Фробениуса, $[b, c]=1$;

5. $G=((\langle z\rangle \times\langle a\rangle) \lambda\langle b\rangle) \lambda\langle c\rangle, z^{p^{2}}=a^{p}=b^{p}=1,[a, b]=z^{p}, w(|c|) \leqslant 3, a^{c}=a^{\alpha}$, 
$b^{c}=b^{\beta}, z^{c}=z^{\alpha \beta}$ и либо $(\alpha)=|c| / r, \wp(\beta)=\wp(\alpha \beta)=|c|$, либо $\wp(\alpha)=|c| / r_{1}$, $\wp(\beta)=|c| / r_{2}, \wp(\alpha \beta)=|c|, r, r_{1}, r_{2}$ - простые делители $|c|, r_{1} \neq r_{2} ;$

6. $G=((\langle a\rangle \times\langle z\rangle) \lambda\langle b\rangle) \lambda\langle c\rangle, a^{p^{2}}=b^{p}=z^{p}=1,[a, b]=z, w(|c|) \leqslant 3, a^{c}=a^{\alpha}$, $b^{c}=b^{\beta}, z^{c}=z^{\alpha \beta}, \wp(\alpha)=\wp(\alpha \beta)=|c|, \wp(\beta)=|c| / r, r-$ простое число;

7. $G=((\langle a\rangle \times\langle b\rangle) \lambda\langle x\rangle) \lambda\langle c\rangle, a^{p^{2}}=b^{p}=x^{p}=1,[a, x]=b,[b, x]=a^{s p}, s=1$ или квадратичный невьчет по модулю $p, w(|c|)=1,[x, c]=1,\langle a\rangle \lambda\langle c\rangle u$ $\langle b\rangle \lambda\langle c\rangle-$ zруппы Фробениуса;

8. $G=((\langle z\rangle \times\langle a\rangle \times\langle b\rangle) \lambda\langle x\rangle) \lambda\langle c\rangle, z^{p}=a^{p}=b^{p}=x^{p}=1,[b, x]=a,[a, x]=z$, $[z, x]=1, w(|c|)=2, z^{c}=z^{\alpha}, a^{c}=a^{\alpha}, b^{c}=b^{\alpha}, x^{c}=x, \wp(\alpha)=|c| ;$

9. $G=((\langle z\rangle \times\langle a\rangle) \lambda\langle b\rangle) \lambda\langle c\rangle, z^{p}=a^{p}=b^{p}=1,[a, b]=z, a^{c}=a^{\alpha}, b^{c}=b^{\beta}$, $z^{c}=z^{\alpha \beta}$ и выполняется один из следуюших случаев:

a) $w(|c|)=2, \alpha=1, \wp(\beta)=|c|$;

б) $w(|c|)=3, \wp(\alpha)=r$ - простое число, $(|c| / r, r)=1, \wp(\beta)=|c| / r$ или $|c|$, $\wp(\alpha \beta)=|c|$;

в) $w(|c|) \leqslant 3, \wp(\alpha)=|c| / m, \wp(\beta)=|c|, \wp(\alpha \beta)=|c| / r, r-$ nростое число, $(m, r)=1$

г) $w(|c|)=3, \wp(\alpha)=|c| / r_{1}, \wp(\beta)=|c| / r_{2}, \wp(\alpha \beta)=|c| / r_{3}, r_{1}, r_{2}, r_{3}$ - различные простые числа;

10. $G=(\langle a\rangle \lambda\langle b\rangle) \lambda\langle c\rangle, a^{p^{2}}=b^{p}=1,[a, b]=a^{p},[b, c]=1, w(|c|)=2,\langle a\rangle \lambda\langle c\rangle-$ группа Фробениуса;

11. $G=((\langle z\rangle \times\langle a\rangle) \lambda\langle b\rangle) \lambda(\langle c\rangle \times\langle d\rangle), a^{p}=b^{p}=z^{p}=1,[a, b]=z,[z, c]=1$, $a^{c}=a^{\alpha} b^{\beta} z^{-\alpha \beta / 2}, b^{c}=a^{\gamma} b^{\delta} z^{-\gamma \delta / 2}, \beta \gamma \neq 0, \alpha \delta-\beta \gamma=1, a^{d}=a^{s}, b^{d}=b^{s}$, $z^{d}=z^{s^{2}},|c|$ - нечетный простой делитель числа $(p+1),|d|$ нечетен, $w(|d|) \leqslant 2, \wp(s)=|d|$.

ДокАЗАтЕльство. Пусть $G$-группа из условия теоремы. Тогда $|F| \in\left\{p^{3}, p^{4}, p^{3} q\right\}$, $p$ и $q$ - различные простые числа. Так как $Z(F) \cap F^{\prime}$ - циклическая нормальная в группе $G$ подгруппа простого порядка, то $F$ является холловой подгруппой группы $G$. Поэтому $G=F \lambda K,(|F|,|K|)=1$. Если $G$ - группа Фробениуса, то $G$ - группа типа 1$)$. Поэтому будем считать, что $K$ действует на $F$ нерегулярно.

Предположим сначала, что $|F|=p^{3} q$. Тогда $F=P \times\langle x\rangle, P$ - неабелева группа порядка $p^{3},|x|=q, G=F \lambda\langle c\rangle,|c|$ делит $(p-1, q-1)$ и $w(|c|) \leqslant 4$. Пусть $a$ и $c_{0}$ такие неединичные элементы из $F$ и $\langle c\rangle$ соответственно, что $\left[a, c_{0}\right]=1$. Тогда из $\langle a\rangle \cap Z(F)=1$ следует, что $|a|=p$ и $w(|c|) \leqslant 3$. Кроме того, из $a^{c} \in C\left(c_{0}^{c}\right)=C\left(c_{0}\right)$ следует, что подгруппа $\langle a\rangle c$-допустима.

Рассмотрим сначала случай, когда $P$ - групша экспоненты $p$, т.е.

$$
P=(\langle z\rangle \times\langle a\rangle) \lambda\langle b\rangle, \quad a^{p}=b^{p}=z^{p}=1, \quad[a, b]=z .
$$

Положим $H=P \lambda\left\langle c_{0}\right\rangle$. И пусть $z^{c_{0}}=z^{k}, b^{c_{0}}=z^{\alpha} a^{\beta} b^{\gamma}$. Если $\gamma=1$, то $\left[b, c_{0}\right] \in\langle z\rangle \times\langle a\rangle$ и из $P=\left[P, c_{0}\right] \cdot C_{P}\left(c_{0}\right)$ получим, что $C\left(c_{0}\right)$ содержит элемент вида $z^{s} a^{t} b$. Но тогда $C\left(c_{0}\right)$ содержит и $z=\left[a, z^{s} a^{t} b\right]$, что невозможно. Поэтому $\gamma \neq 1$. Заменяя $b$ на $a^{r} b$, где $r(\gamma-1) \equiv \beta(p)$, будем иметь

$$
\left(a^{r} b\right)^{c_{0}}=a^{r} z^{\alpha} a^{\beta} b^{\gamma}=z^{\alpha} a^{r+\beta} b^{\gamma} \equiv\left(a^{r} b\right)^{\gamma}(\bmod \langle z\rangle)
$$


т.е. можно считать, что $\beta=0$. Из равенств

$$
z^{k}=z^{c_{0}}=[a, b]^{c_{0}}=\left[a, b^{c_{0}}\right]=\left[a, z^{\alpha} b^{\gamma}\right]=z^{\gamma}
$$

получим $k=\gamma$. Применяя к $(\langle z\rangle \times\langle b\rangle) \lambda\left\langle c_{0}\right\rangle$ теорему Машке, можно считать, что $b^{c_{0}}=b^{k}$. Если $C(b) \cap\langle c\rangle \neq 1$, то, очевидно, $\left(|C(b) \cap\langle c\rangle|,\left|c_{0}\right|\right)=1$.

Итак, если $P$ имеет экспоненту $p$, то $G$ - групша типа 2$)$. Пусть теперь

$$
P=\langle b\rangle \lambda\langle a\rangle, \quad b^{p^{2}}=a^{p}=1, \quad[a, b]=b^{p} .
$$

Так как число циклических подгрупп порядка $p^{2}$ в группе $P$ равно $p$, а $|c|$ делит $p-1$, то можно считать, что подгруппа $\langle b\rangle$-допустима. Таким образом, $\langle b\rangle \lambda\langle c\rangle$ и $\langle x\rangle \lambda\langle c\rangle-$ группы Фробениуса. Как и выше, подгруппа $\langle a\rangle$ тоже $c$-допустима. Пусть $a^{c}=a^{n}$ и $b^{c}=b^{\alpha}$. Тогда

$$
b^{p \alpha}=\left(b^{p}\right)^{c}=[a, b]^{c}=\left[a^{c}, b^{c}\right]=b^{p n \alpha} .
$$

Отсюда $n \equiv 1(p), c \in C(a)$ и $G$ - группа типа 3 .

Предположим теперь, что $|F|=p^{4}$. Тогда $G=F \lambda\langle c\rangle$ и $|c|$ делит число $(p-1)$. Полньй список групш порядка $p^{4}$ приведен, например, в [7, III.12.6]. Пусть $|Z(F)|=p^{2}$. Тогда $F$ одна из следующих групш:

a) $F=P \times\langle x\rangle, P$ - неабелева группа порядка $p^{3}$;

б) $F=\langle a\rangle \lambda\langle b\rangle, a^{p^{2}}=b^{p^{2}}=1,[a, b]=a^{p}$;

в) $F=\langle a\rangle \lambda\langle b\rangle, a^{p^{3}}=b^{p}=1,[a, b]=a^{p^{2}}$;

г) $F=(\langle z\rangle \times\langle a\rangle) \lambda\langle b\rangle, z^{p^{2}}=a^{p}=b^{p}=1,[a, b]=z^{p},[b, z]=1$;

д) $F=(\langle a\rangle \times\langle b\rangle) \lambda\langle x\rangle, a^{p^{2}}=b^{p}=x^{p}=1,[a, x]=b,[b, x]=1$.

В случае а) мы снова получим группы типа 2) или 3 ) при $q=p$. Случай б) невозможен. Рассмотрим случай в). Здесь, как и выше, можно считать, что $a^{c}=a^{\alpha}, b^{c}=b^{\beta}$ и $C(b) \cap\langle c\rangle \neq 1 . И_{3}$

$$
a^{p^{2} \alpha}=\left(a^{p^{2}}\right)^{c}=[a, b]^{c}=\left[a^{c}, b^{c}\right]=a^{p^{2} \alpha \beta}
$$

получаем, что $\beta \equiv 1(p)$, т.е. $|c|-$ простое число и $G$ - группа типа 4 .

Если $F$ типа г), то $\langle z\rangle \lambda\langle c\rangle$ - группа Фробениуса и можно считать, что $C(a) \cap\langle c\rangle \neq 1$. Как и выше, несложно показать, что $G$ - группа типа 5). Наконец, в случае д) имеем $(\langle a\rangle \times\langle b\rangle) \lambda\langle c\rangle-$ группа Фробениуса и $G-$ группа типа 6$)$.

Предположим теперь, что $|Z(F)|=p$. В этом случае $F$ - одна из следующих групп:

a) $F=(\langle a\rangle \times\langle b\rangle) \lambda\langle x\rangle, a^{p^{2}}=b^{p}=x^{p}=1,[a, x]=b,[b, x]=a^{s p}$, где $s=1$ или $s-$ квадратичньй невычет по модулю $p$;

б) $F=(\langle z\rangle \times\langle a\rangle \times\langle b\rangle) \lambda\langle x\rangle, z^{p}=a^{p}=b^{p}=x^{p}=1,[z, x]=1,[a, x]=z,[b, x]=a$;

в) $F=(\langle a\rangle \lambda\langle b\rangle) \lambda\langle x\rangle, a^{p^{2}}=b^{p}=x^{p}=1,[a, b]=a^{p},[a, x]=b,[b, x]=1$ при $p>3$, и $F=(\langle a\rangle \times\langle b\rangle)\langle x\rangle, a^{9}=b^{3}=1, a^{3}=x^{3},[a, x]=b,[b, x]=a^{3}$ при $p=3$.

Рассмотрим случай а). Предположим сначала, что $\langle c\rangle$ действует на $Z_{2}(F)=\langle a\rangle \times\langle b\rangle$ нерегулярно. Тогда можно считать, что $\langle a\rangle \lambda\langle c\rangle$ - группа Фробениуса и $C(b) \cap\langle c\rangle=$ $\left\langle c_{0}\right\rangle \neq 1$. Положим $a^{c_{0}}=a^{\alpha}, x^{c_{0}}=a^{p \beta} b^{\gamma} x^{\delta}$. Если $\delta=1$, то, как и выше, получим противоречие вида $a^{p} \in C\left(c_{0}\right)$. Поэтому $\delta \neq 1$. Из

$$
b=b^{c_{0}}=\left[a^{c_{0}}, x^{c_{0}}\right]=\left[a^{\alpha}, a^{p \beta} b^{\gamma} x^{\delta}\right]=b^{\alpha \delta} a^{s p \alpha \delta(\delta-1) / 2}
$$


имеем $\alpha \delta \equiv 1(p)$ и $s \alpha \delta(\delta-1) / 2 \equiv 0(p)$, что невозможно.

Если $\langle c\rangle$ действует на $Z_{2}(F)$ регулярно, то можно считать, что $a^{c}=a^{\alpha}, b^{c}=b^{\beta}$ и $x^{c}=x^{\gamma}$. Поэтому

$$
b^{\beta}=b^{c}=[a, x]^{c}=\left[a^{c}, x^{c}\right]=\left[a^{\alpha}, x^{\gamma}\right]=b^{\alpha \gamma} a^{s p \alpha \gamma(\gamma-1) / 2} .
$$

Отсюда $\alpha \gamma \equiv \beta(p)$ и $\gamma=1$, т.е. $G$ - группа типа 7 .

Пусть $F$ типа б) и $\langle c\rangle \cap C(a)=\left\langle c_{0}\right\rangle \neq 1$. Положим $z^{c_{0}}=z^{\alpha}, b^{c_{0}}=b^{\gamma}, x^{c_{0}}=a^{u} b^{v} z^{w} x^{\delta}$. Тогда

$$
a=[b, x]^{c_{0}}=\left[b^{\gamma}, a^{u} b^{v} z^{w} x^{\delta}\right]=\left[b^{\gamma}, x^{\delta}\right]=a^{\gamma \delta} z^{\gamma \delta(\delta-1) / 2},
$$

что снова приводится к противоречию.

Аналогично получим противоречие и в случае $\langle c\rangle \cap C(b) \neq 1$. Поэтому можно считать, что $\langle c\rangle \cap C(x) \neq 1$. Так как $\langle z\rangle,\langle z\rangle \times\langle a\rangle=Z_{2}(F),\langle z\rangle \times\langle a\rangle \times\langle b\rangle=C\left(Z_{2}(F)\right)$ $c$-допустимы, то можно считать, что $a^{c}=a^{\alpha}, b^{c}=b^{\beta}, z^{c}=z^{\gamma}, x^{c}=x^{\delta}$. Тогда из $[a, x]=z$ и $[b, x]=a$ получим $\delta=1$ и $\alpha=\beta=\gamma$, т.е. $G-$ грушпа типа 8.

Если $F$-групша типа в) то и в случае $\langle c\rangle \cap C(b) \neq 1$, и в случае $\langle c\rangle \cap C(x) \neq 1$ получим противоречие.

Рассмотрим теперь случай $|F|=p^{3}$. Если $C(Z(F))=F$, то аналогично предыдущему получим групп типа $9 \mathrm{a}), 9 б)$ или 10$)$. Поэтому остается рассмотреть случай $C(Z(F))>F$.

Пусть $G=F \lambda K, C(Z(F))=F \lambda\langle c\rangle$. Если

$$
F=\langle a\rangle \lambda\langle b\rangle, \quad a^{p^{2}}=b^{p}=1, \quad[a, b]=a^{p},
$$

то можно считать, что $a^{c}=a$. Если $b^{c}=a^{p \alpha} b^{\beta}$, то $a^{p}=\left(a^{p}\right)^{c}=[a, b]^{c}=a^{p \beta}$, т.е. $\beta=1$. Но тогда $C(Z(F))=F \times\langle c\rangle$, что противоречит выбору подгруппы $F$. Поэтому $F-$ группа экспоненты $p$.

Положим $F=(\langle z\rangle \times\langle a\rangle) \lambda\langle b\rangle, a^{p}=b^{p}=z^{p}=1,[a, b]=z, a^{c}=a^{\alpha} b^{\beta} z^{n}$, $b^{c}=a^{\gamma} b^{\delta} z^{m}$. Тогда $\alpha \delta-\beta \gamma=1$. Если $\langle c\rangle$ действует на $F /\langle z\rangle$ нерегулярно, то снова получим противоречие с выбором подгруппы $F$.

Предположим, что $\langle c\rangle$ действует на $F /\langle z\rangle$ неприводимо. Тогда $|c|$-нечетньй простой делитель числа $(p+1)$. Так как $|K /\langle c\rangle|$ делит $(p-1)$, то $K=\langle c\rangle \lambda\langle d\rangle$. Группа $\langle d\rangle$ действует на $F /\langle z\rangle$ приводимо. Поэтому можно считать, что $a^{d}=a^{s}, b^{d}=b^{t}$ и $z^{d}=z^{s t}$. Но тогда

$$
a^{c d}=\left(a^{\alpha} b^{\beta} z^{n}\right)^{d}=a^{s \alpha} b^{t \beta} z^{n s t}
$$

и

$$
a^{d c}=\left(a^{s}\right)^{c}=a^{s \alpha} b^{s \beta} z^{n s-\alpha \beta s(s-1) / 2} .
$$

Отсюда $s=t$ и $n=-\alpha \beta / 2$. Аналогично из $b^{c d}=b^{d c}$ получим $m=-\gamma \delta / 2$. Так как $s=t$, то $z^{d}=z^{t^{2}}$ и из $\wp\left(t^{2}\right)=|d|$ следует, что $|d|$ нечетен, т.е. $G$-группа типа 11$)$.

Предположим теперь, что $c d \neq d c$. Тогда [7, II.7.3] можно считать, что $d^{2} \in C(c)$. Пусть $|d|=2 m$. Так как $d^{2} c=c d^{2}$, то по уже доказанному число $m$ нечетно и $s^{2}=t^{2}$. Из $z^{d}=\left[a^{d}, b^{d}\right]=z^{s t}$ следует, что $\wp(s t)=2 m$. В этом случае групша $\langle c\rangle \lambda\langle d\rangle$ действует на $F /\langle z\rangle$ регулярно и в то же время содержит нециклическую подгруппу $\langle c\rangle \lambda\left\langle d^{m}\right\rangle$ порядка $2|c|$, что противоречит строению регулярных групп автоморфизмов. 
Если же $c$ действует на $F /\langle z\rangle$ приводимо, то можно считать, что

$$
a^{c}=a^{\alpha}, \quad b^{c}=b^{\beta}, \quad \alpha \beta=1 \quad \text { и } \quad \alpha \neq 1 .
$$

Рассматривая действие подгрупшы $K$ на фактор-группе $F /\langle z\rangle$, можно отождествить ее с подгруппой из $G L(2, p)$. Тогда из $K \leqslant N(\langle c\rangle)$ следует, что если $K$ неабелева, то $K \leqslant D \lambda\langle\tau\rangle$, где $D$ - группа диагональных матриц, $a^{\tau}=b, b^{\tau}=a$.

Если подгруппа $K=\langle d\rangle$ циклическая, то в зависимости от условий $C(a) \cap K=$ $C(b) \cap K=1, C(a) \cap K \neq 1=C(b) \cap K$ и $C(a) \cap K \neq 1 \neq C(b) \cap K$ получим группы 9 в) при $m=1$ и $m \neq 1$ или 9 г).

Пусть $K=\langle c\rangle \times\langle d\rangle$ - абелева нециклическая подгруппа и $\langle c\rangle \times\left\langle d_{0}\right\rangle$ элементарная абелева порядка $|c|^{2}$. Тогда [12, с. 225]

$$
\left.F=\left\langle C_{F}(x)\right|\left(\langle c\rangle \times\left\langle d_{0}\right\rangle\right) /\langle x\rangle-\text { циклическая }\right\rangle .
$$

Если $\alpha \neq-1$, то $\langle d\rangle$ диагональна. Если же $\alpha=-1$, то в силу (*) и теоремы Машке можно считать, что $\left\langle d_{0}\right\rangle$ диагональна.

Положим $a^{d_{0}} \equiv a, b^{d_{0}} \equiv b^{t}(\bmod \langle z\rangle)$ и $c^{n} d_{0} \in C\left(a^{m} b z^{k}\right)$. Тогда из

$$
a^{m} b z^{k}=\left(a^{m} b z^{k}\right)^{d_{0}} \equiv a^{m \alpha^{n}} b^{t \beta^{n}}(\bmod \langle z\rangle)
$$

следует, что $t=\beta^{-n}=\alpha^{n}$ и $\alpha^{n}=1$. Но тогда $t=1$, что невозможно.

Если $K$ неабелева, то, как и вьше, получим противоречие со строением регулярных групп автоморфизмов.

\section{СПИСОК ЦИТИРОВАННОЙ ЛИТЕРАТУРЫ}

[1] Bianchi M., Manz O. Groups with small centralizers of non-central elements // Boll. Un. Mat. Ital. 1990. V. 4A. № 7. P. 365-370.

[2] Scarselli A. Gruppi con piccoli centralizzanti // Boll. Un. Mat. Ital. 1992. V. 6B. №7. P. 649-663.

[3] Schmidt R. Zentralisatorverbände endlicher Gruppen // Rend. Sem. Math. Univ. Padova. 1970. № 44. P. 97-131.

[4] Васильева А. В. О централизаторных решетках конечных простых групп // Сиб. матем. ж. 1997. T. 18. № 2. C. 263-273.

[5] Bloom D. M. The subgroups of $P S L(3, q)$ for odd $q / /$ Trans. Amer. Math. Soc. 1967. V. 127. P. 150-178.

[6] Гаген Т. М. Некоторые вопросы теории конечных групп // К теории конечных групп. М.: Мир, 1979.

[7] Huppert B. Endliche Gruppen. V. 1. Berlin-Heidelberg-New York: Springer-Verlag, 1967.

[8] Brauer R., Suzuki M. On finite groups of even order whose 2-Sylow group is a quaternion group // Proc. Nat. Acad. Sci. USA. 1959. V. 45. № 12. P. 1757-1759.

[9] Suzuki M. Two characteristic properties of ZT-groups // Osaka Math. J. 1963. V. 15. P. 143-150.

[10] Бусаркин В. М., Горчаков Ю. М. Конечные расщепляемые группы. М.: Наука, 1968.

[11] Супруненко Д. А. Группы матриц. М.: Наука, 1972.

[12] Gorenstein D. Finite Groups. New York: Harper and Row, 1968. 